\title{
Development of a Multi-Event Trajectory Optimization Tool for Noise-Optimized Approach Route Design
}

\author{
M.L Braakenburg ${ }^{1}$, S. Hartjes ${ }^{2}$ and H.G. Visser ${ }^{3}$ \\ Delft University of Technology, Delft, the Netherlands \\ S.J. Hebly ${ }^{4}$ \\ National Aerospace Laboratory, Amsterdam, The Netherlands
}

\begin{abstract}
This paper presents preliminary results from an ongoing research effort towards the development of a multi-event trajectory optimization methodology that allows to synthesize RNAV approach routes that minimize a cumulative measure of noise, taking into account the total noise effect aggregated for all inbound flight movements taking place within an operational year. This new development is an extension of a tool called NOISHHH which was developed earlier for the synthesis of single-event noise abatement RNAV trajectories into and out of airports. Although the presented numerical examples pertain to a specific airport in the Netherlands, viz. Rotterdam the Hague airport, this study focuses on the development of a generic methodology that can be applied to any given airport. Initial application of the adapted optimization framework to the design of noise abatement RNAV approach routes at Rotterdam The Hague airport reveals a significant potential for reducing the number of people highly annoyed due to annual noise exposure relative to the existing situation.
\end{abstract}

\section{Introduction}

$\mathrm{T}$ he strong growth in commercial air traffic and the rising level of urbanization around airports over the past decades has led to an increased level of noise nuisance in communities surrounding airports. One of the approaches to reduce the impact of air traffic noise on local communities is to design noise friendly departure and arrival routes and procedures. In response to the need for more environmentally friendly flight paths, a tool named NOISHHH has been developed at the TU Delft that enables to generate routings and flight-paths for both inbound and outbound flights that minimize the environmental impact in the residential communities surrounding the airport, while satisfying all imposed operational and safety constraints ${ }^{1-3}$. The most recent version of NOISHHH offers the possibility to calculate noise-optimized RNAV routes that can be programmed into the Flight Management System (FMS) currently available in most aircraft cockpits ${ }^{4,5}$. It must be noted though that the NOISHHH tool only considers single event flyovers and does not assess the community noise impact based on an aggregation over all aircraft operations for a particular time period. Indeed, a trajectory generated using NOISHHH relates to a single flight event only and is specific for each aircraft type and flight condition (e.g., aircraft gross weight) considered. Unfortunately, however, assigning each flight its own individually optimized departure or approach route does not comply with current operational practice. Moreover, the impact of airport noise on the community is typically quantified based on an aggregation over all aircraft operations for a particular time period (e.g., a year) as opposed to the effect of isolated events. For this reason the TU Delft has embarked on a research effort to develop a multievent version of NOISHHH that seeks to synthesize RNAV routes that minimize a cumulative measure of noise,

\footnotetext{
${ }^{1}$ MSc student

${ }^{2} \mathrm{PhD}$ candidate

${ }^{3}$ Associate Professor, Faculty of Aerospace Engineering; Associate Fellow AIAA.

${ }^{4} \mathrm{PhD}$ candidate; R\&D engineer, Environment and Policy Support.
} 
taking into account the total noise effect of all flight movements over a specified period. The usefulness of the new tool is demonstrated in numerical examples pertaining to the synthesis of RNAV approach routes to Rotterdam The Hague airport in the Netherlands.

Although numerous studies related to the theoretical development of optimal trajectories that minimize the noise impact in departure or approach trajectories have appeared in the literature in recent years ${ }^{6-8}$, none of them specifically deal with the synthesis of trajectories that aim to minimize the aggregate, long term noise impact. Several studies have been conducted that were aimed at minimizing a cumulative noise metric that aggregates the effects of all aircraft movements to and from the airport for a specified period of time ${ }^{9-12}$, however, these optimization studies typically relate to resource allocation problems, in which individual aircraft movements are optimally allocated to routes that are selected from a set of available pre-defined routes. In the open literature only a single study has been found that distinctly deals with multi-event environmental trajectory optimization formulation $^{13}$. In this study, however, the focus is essentially on conflict resolution in an environmentally responsible manner and the number of concurrent aircraft movements considered therefore remains limited.

The flight path of an aircraft can be decomposed into two components, the ground track (route) and the vertical profile (i.e., speed/altitude/thrust profiles). In this work, the primary goal is to optimally shape the shared ground track, assuming given procedures (altitude and speed profiles as functions of distance to runway) for the various aircraft types that visit Rotterdam The Hague airport within the considered operational year. In this study, the prescribed vertical profiles have been extracted from the guidelines for noise calculations in the Netherlands ${ }^{14}$. In these guidelines, aircraft types are grouped into acoustic categories and for each category the speed and altitude profiles to be used in the computation of the noise footprint of arrival trajectories are explicitly specified. Each category is modeled using a single, acoustically representative, aircraft type. In the case of Rotterdam The Hague airport, the annual traffic can be quite well represented with just three aircraft categories. With the vertical profiles prescribed for each aircraft type, the collective trajectory optimization problem effectively only addresses the ground track of a single approach route shared by all aircraft.

In this paper, we first formulate the design of a community noise optimized approach route as a multi-event trajectory optimization problem. Next, a brief discussion on the employed trajectory optimization technique is presented. The overall approach is then demonstrated in two numerical examples involving the design of community noise optimized RNAV approach routes to runway 24 of Rotterdam The Hague airport. Finally, we present conclusions and directions for future research.

\section{Multi-event trajectory optimization formulation}

The aircraft trajectory optimization framework developed in this study relies on a point-mass dynamic model formulation. With the vertical profiles prescribed for each aircraft type, the trajectory optimization problem in essence only needs to address the ground track kinematics shared by all aircraft. Using along-track distance $s$ as the independent variable, and assuming small flight path angle $\gamma$ such that $\cos (\gamma) \approx 1$, the kinematic equations can be written as:

$$
\begin{aligned}
& \frac{d x}{d s}=\cos \chi(s) \\
& \frac{d y}{d s}=\sin \chi(s) \\
& \frac{d \chi}{d s}=u(s),
\end{aligned}
$$

where $\chi(s)$ is the heading angle and $x$ and $y$ are the position coordinates of the aircraft in a Cartesian reference frame. Note that a standard atmosphere and a no-wind condition have been assumed here. The heading rate $u$ serves as the control variable in the optimal control formulation. Note that the heading rate $u$ is simply the reciprocal of the turn radius $R$, i.e. $u=1 / R$. By using the heading rate $u$, rather than the heading angle $\chi$ as the control variable, operational constraints such as turn rate and bank angle limits can be readily enforced in the problem formulation. 
Combining the drag polar of each aircraft type considered with the prescribed speed profile $V(s)$, altitude profile $h(s)$ and the optimal control history $\chi(s)$, enables the assessment of the bank angle $\mu(s)$ and subsequently the required thrust $T(s)$ from the relations:

$$
\begin{aligned}
& \tan \mu(s)=u(s) \cdot \frac{V(s)^{2}}{g_{0}} \\
& T(s)=V(s) \cdot \frac{d V}{d s}(s) \cdot \frac{W}{g_{0}}+\frac{1}{2} \rho(s) V(s)^{2} S C_{D}+W \cdot \frac{d h}{d s}(s),
\end{aligned}
$$

where $C_{D}$ is the drag coefficient, and $W$ the aircraft gross weight. With the thrust levels needed to fly the optimal trajectory now known, the sound exposure levels (SEL) at specified locations around the airport can be calculated. For the acoustic calculations, use is made of the well-known Integrated Noise Model INM ${ }^{15}$. INM has been the FAA's standard tool for airport noise assessments for decades and is probably the most widely used tool for noise assessment throughout the world. The INM can calculate the SEL in any point in a pre-defined grid arranged around the airport, for any aircraft type. The SEL footprint is computed for the arrival trajectory for each of the three aircraft types representing a particular aircraft category ${ }^{14}$. Using historic flight movement data for flights inbound to Rotterdam The Hague airport (recorded from 26-1-2009 26-1-2010) the noise contribution of all individual arrival movements that fly that particular route in a given year can be aggregated into the $L_{d e n}$ cumulative noise metric:

where:

$$
L_{d e n}=10 \log \left[\sum_{i=1}^{N_{A C \text { types }}} n(i) \cdot w(i) \cdot 10^{\frac{S E L(i)}{10}}\right]-10 \log T \quad(d B A),
$$

- $\quad i$ is the aircraft category identification number, which ranges from 1 to $N_{\text {ACtypes}}$, the number of aircraft types considered in the study (here, $N_{\text {ACtypes }}=3$ )

- $\quad n(i)$ represents the number of aircraft of type $i$ that fly the arrival route over a period of one year.

- $w(i)$ represents the average (day/night/evening) noise penalty factor over a period of one year, associated with aircraft type $i$.

- $\quad \operatorname{SEL}(i)$ defines the SEL value at the considered location corresponding to aircraft type $i$.

- $\quad T$ is the averaging period, which for practical applications may be considered to be one year: $T=365 \cdot 24 \cdot 3600=31536000$ seconds.

Using a dose-response relationship that couples the $L_{d e n}$ value in a grid cell to a percentage of people being highly annoyed, in conjunction with the actual population density distribution in the grid area, an estimate can be made of the total number of people expected to be highly annoyed by aircraft noise on an annual basis for the considered route.

The particular dose-response relationship that couples the $L_{d e n}$ value to a certain level of human annoyance employed is this study was originally conceived by the Dutch organizations TNO and RIVM (National Institute for Public Health and the Environment) ${ }^{16}$. The dose-response relationship as proposed by TNO and RIVM has been determined based on a large scale research effort conducted in 2002, which focused on the way aircraft noise was experienced by a representative part of the population ${ }^{16}$. This relationship, shown in Figure 1, can be expressed as:

$$
\% P H=\left(\frac{e^{-8.11001+0.1333 \cdot L_{d e n}}}{1+e^{-8.11001+0.1333 \cdot L_{d e n}}}\right) \cdot 100,
$$

where $\% P H$ is the percentage of the population to be highly annoyed by aircraft noise due to a certain $L_{d e n}$ value. By multiplying this percentage with the amount of people living in a particular grid cell, the amount of people highly annoyed can be determined for that cell. Finally, by summing the amount of people highly annoyed over all grid cells, the total number of people highly annoyed $P H$ can be determined. In this study, the total number of people highly annoyed $\mathrm{PH}$ serves as the primary environmental performance criterion.

The main objective of this study is to design routes that minimize the noise impact. However, noise impact is certainly not the only consideration of importance. Indeed, to avoid excessive airline economic and air quality penalties due to path extension, a secondary performance index has been included in the overall objective function. 
More specifically, a weighted combination of the number of people highly annoyed and the overall path length of the approach route has been considered:

$$
J=k_{\text {noise }} \cdot P H+k_{\text {distance }} \cdot s_{f},
$$

where $k_{\text {noise }}$ and $k_{\text {distance }}$ are the weighting factors of the number of people highly annoyed $P H$ and the total route distance $s_{f}$. It is noted that for a given speed profile as a function of distance to runway $\left(s_{f}-s\right)$, minimizing the total route distance boils down to minimizing the total flight time. As an alternative to the path length criterion, average fuel consumption could be considered. However, this choice is likely to result in a higher computational burden. Including fuel considerations in the composite performance index is an issue of future research interest though.

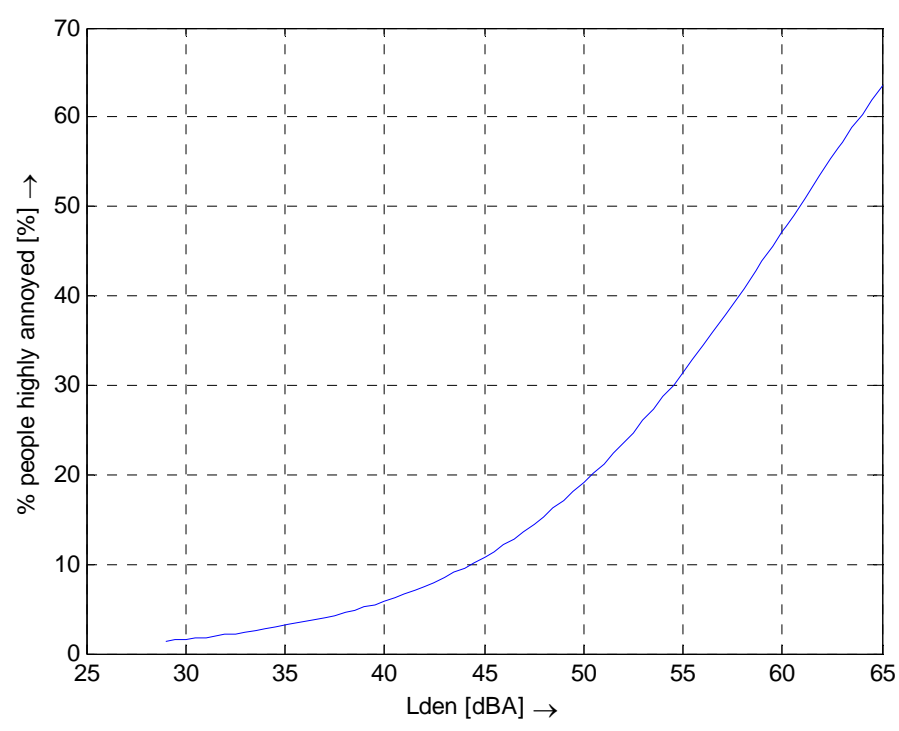

Figure 1. Dose-response relationship between $L_{d e n}$ and the percentage of people Highly Annoyed $\% P H$ (Source: Ref.16).

For a realistic problem formulation a number of operational constraints must be taken into account. The two most important constraints that need to be included to obtain optimized trajectories that meet the operational and safety requirements are a turn rate constraint (in deg./s) and a bank angle constraint. The rate of turn in deg./s is defined as:

$$
\frac{d \chi}{d t}=\frac{d \chi}{d s} \cdot \frac{d s}{d t}=u(s) \cdot V(s)
$$

For a given turn rate limit in deg./s, Eq.(9) places a constraint on the permissible range for the control $u(s)$ :

$$
|u(s)| . V(s) \leq\left[\frac{d \chi}{d t}\right]_{\max } \Rightarrow|u(s)| \leq \frac{\left[\frac{d \chi}{d t}\right]_{\max }}{V(s)}
$$

Similarly, a bank angle constraint results in the following limitation on the control $u(s)$ :

$$
|\tan \mu(s)|=|u(s)| \cdot \frac{V(s)^{2}}{g_{0}} \leq \tan \mu_{\max } \Rightarrow|u(s)| \leq \frac{\tan \mu_{\max }}{V(s)^{2}} g_{0} \quad
$$


where Eq.(4) was used. It is noted that both control constraints (10) and (11) are speed dependent. Since the speed profile $V(s)$ varies per aircraft type, it is readily clear that the aircraft type that features the highest speed at a given location $s$ imposes the severest limitation and thus represents the most critical constraint at that location. In Figure 2 the constraints (10) and (11) are graphically represented for the values of the bank angle and turn rate limit assumed in this study. It can be seen that for speeds in excess of $170 \mathrm{kts}$, the bank angle constraint overrules the standard rate turn constraint. For speeds lower than $170 \mathrm{kts}$, the standard rate turn constraint determines the maximum allowable value of $u(s)$.

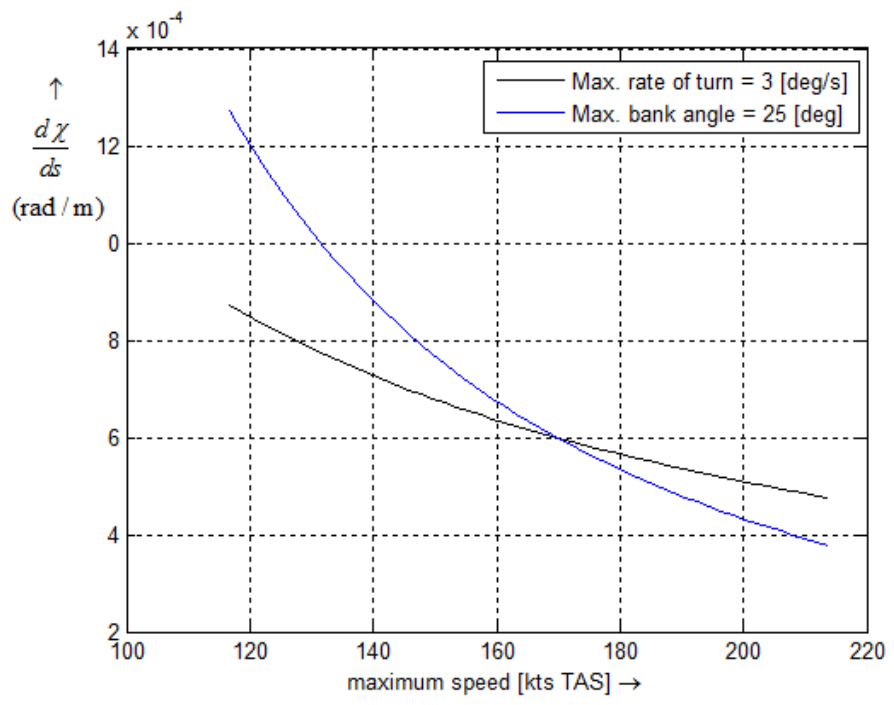

Figure 2. Variation of the maximum allowable value of the control $u(s)$ according to the maximum bank angle constraint and the standard rate turn constraint.

In addition to the turn rate and bank angle constraints (10) and (11), the new version of the NOISHHH tool also features the possibility to (optionally) include constraints associated to the implementation for RNAV routes. An RNAV route can be loosely defined as a sequence of lateral directives along a set of waypoints, enabling the construction of a horizontal flight path between waypoints that can be pre-programmed and automatically executed using the FMS technology currently available in most aircraft ${ }^{6}$.

An RNAV approach route (horizontal flight path) is built from one or more segments. Each RNAV route segment contains two basic elements, (i) a waypoint, which is a specific location defined by latitude and longitude coordinates, and (ii) a leg type, which defines the path before, after or between waypoints. For RNAV operations a total of 23 different leg types are available ${ }^{6}$. However, for the design of flight routes only two basic leg types have been implemented in NOISHHH, viz., Track-to-Fix (TF) and Radius-to-Fix (RF). As shown in Figure 3, a TF leg simply connects two waypoints by a straight line. An RF leg defines a constant radius turn between two waypoints with a fixed radius around a given center point. The RF segment connects two waypoints with a curved path that is tangent to adjoining TF segments.

Implementation of the RNAV constraints in the modified NOISHHH version is relatively simple. In view of the fact that:

$$
\frac{d \chi}{d s}=u(s)=\frac{1}{R(s)}
$$

it is readily clear that a TF leg should be flown with $d \chi / d s=0$, while for an RF leg it is simply required that $d \chi / d s=$ $1 / R=$ constant. Note that the turn radius $R$ is specified as a parameter to be optimized within given bounds. 

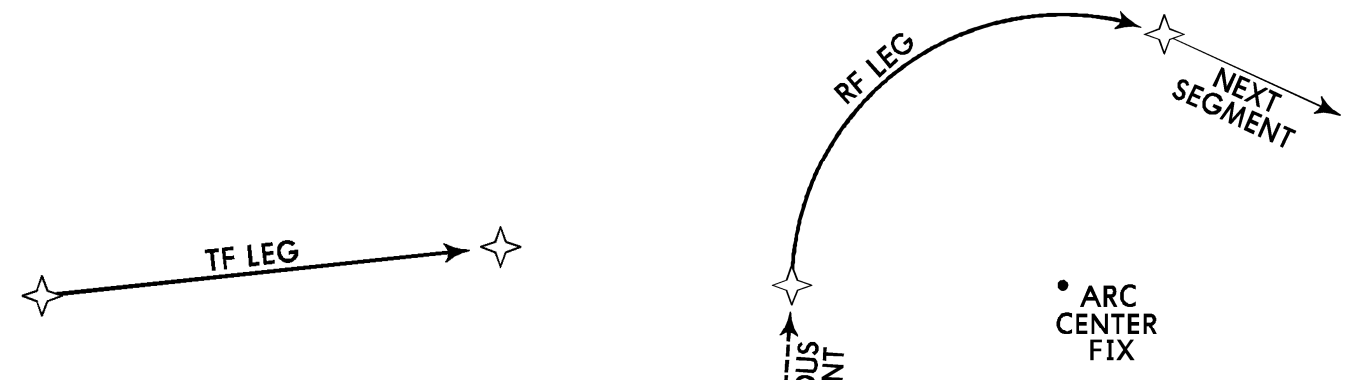

Figure 3. Illustration of RNAV flight segments: TF leg (left) and RF leg (right).

In Figure 4, the various steps needed to calculate a noise-optimized approach route (either RNAV or nonRNAV) within the adapted NOISHHH framework are summarized.

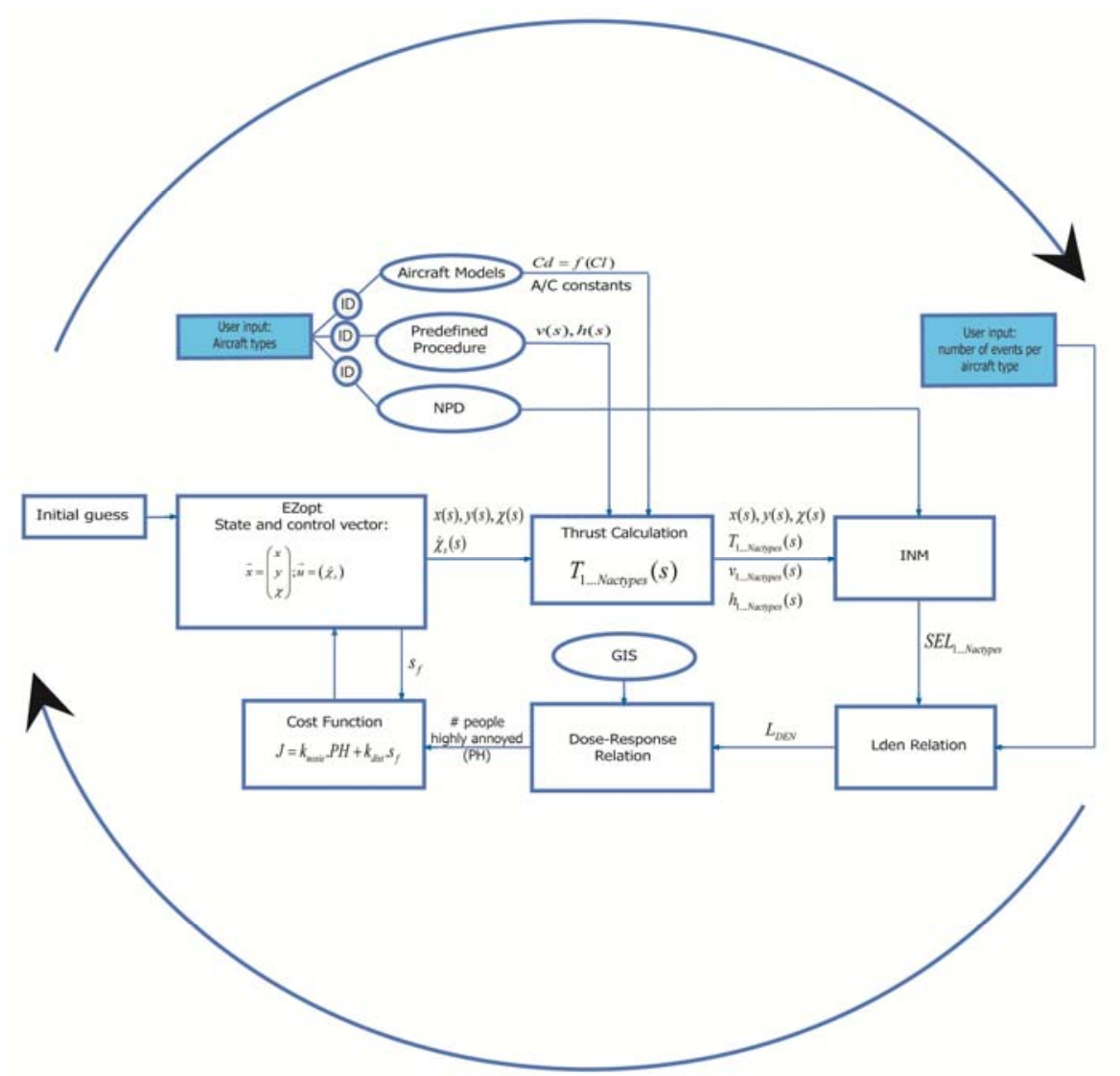

Figure 4. Overview of calculation steps in the modified NOISHHH framework. 


\section{Numerical method for dynamic trajectory optimization}

The core of the NOISHHH tool is the dynamic optimization algorithm. The numerical trajectory optimization method implemented in NOISHHH is the direct optimization technique of collocation with nonlinear programming (NLP). The collocation method essentially transforms an optimal control problem into an NLP formulation by discretizing the trajectory dynamics ${ }^{5}$. To this end, the time interval of an optimal trajectory solution is divided into a number of subintervals. The individual time points delimiting the subintervals are called nodes. The values of the states and the controls at the nodes are then treated as a set of NLP variables. The system differential equations are discretized and transformed into algebraic equations that are enforced at the so-called collocation points which are located in the middle between two nodes (see Figure 5). The path and control constraints imposed in the original optimal control problem are treated as algebraic inequalities in the NLP formulation. To solve the described optimal control problem, a software package called EZopt ${ }^{*}$ has been used. The collocation approach adopted in EZopt results in piecewise constant control histories and piecewise linear state histories. This renders EZopt fully compatible with the discretization (segmentation) approach taken in the INM.
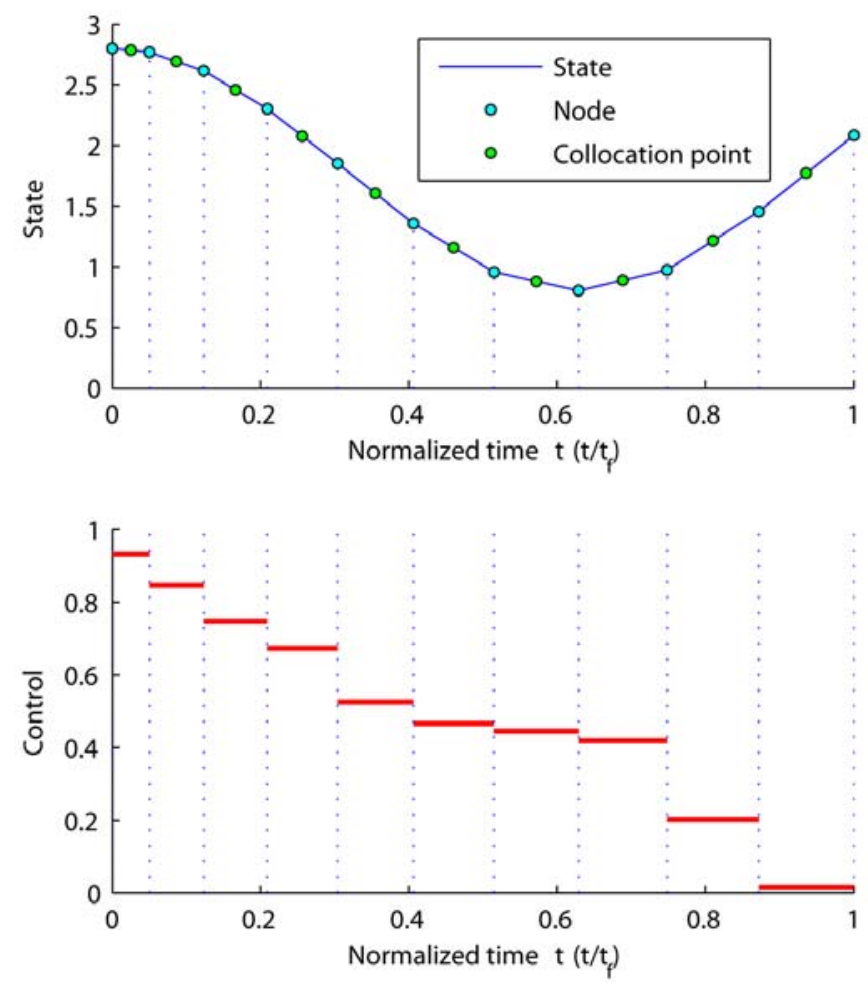

Figure 5. Overview of calculation steps in the modified NOISHHH framework.

Generally, the accuracy of the numerical solution of the problem improves with an increasing number of subintervals, or equivalently, the number of nodes. On the other hand, increasing the number of nodes requires a larger computational effort, hence forcing a compromise between desired accuracy and computational burden. In the NOISHHH context, the adopted flight path segmentation is primarily based on the requirements associated with the computational methodology employed in the INM. The INM includes logic for delimiting the length of path segments. For example, INM subdivides a user-specified path segment if its length multiplied by the change in speed is greater than $100,000 \mathrm{ft}-\mathrm{kts}$. Each solution obtained using NOISHHH is tested for compatibility with the INM segmentation logic

\footnotetext{
${ }^{*}$ http://www.ama-inc.com
} 
The EZopt tool features a so-called multi-phase optimization capability. A multi-phase formulation allows the implementation of different sets of constraints and/or dynamic models for different flight phases. In this study the multi-phase feature has been used to accommodate the RNAV requirements. A multi-phase formulation is indeed needed here since the optimization of RNAV routes requires the trajectory to be divided into several phases, where in each phase the constraints associated to a particular RNAV leg (RF or TF) are implemented.

\section{Numerical Examples}

The capabilities of the new NOISHHH framework are demonstrated in the design of an RNAV noise abatement route in a realistic setting. Figure 6 shows all approaches to Rotterdam The Hague airport for runway 24 for arrivals from the South (here labeled approach 1) and South-West (labeled approach 2) for the year considered (2009). At present, no RNAV approach routes are in place and all inbound flights are essentially vectored by ATC. As shown in Figure 6, this leads to significant track dispersion. It needs to be noted though that this track dispersion is not necessarily detrimental from a cumulative noise perspective.

The flight tracks shown in Figure 6 relate to flights pertaining to just three aircraft categories ${ }^{14}$, each category being represented by a single aircraft type. The three acoustically representative aircraft are, respectively, the Boeing 737-700 (representative of a civil aircraft with 2 turbofan engines and a seating capacity between 100 and 160, CAT 75), the Fokker 50 (representative of a civil aircraft with 2 turboprop engines, CAT 71) and the Cessna Citation II (representative of a small to medium sized business jet, CAT 70). The combined arrival flights of aircraft of these three categories represent more than $80 \%$ of the total arrivals in the considered year for the two approaches shown.

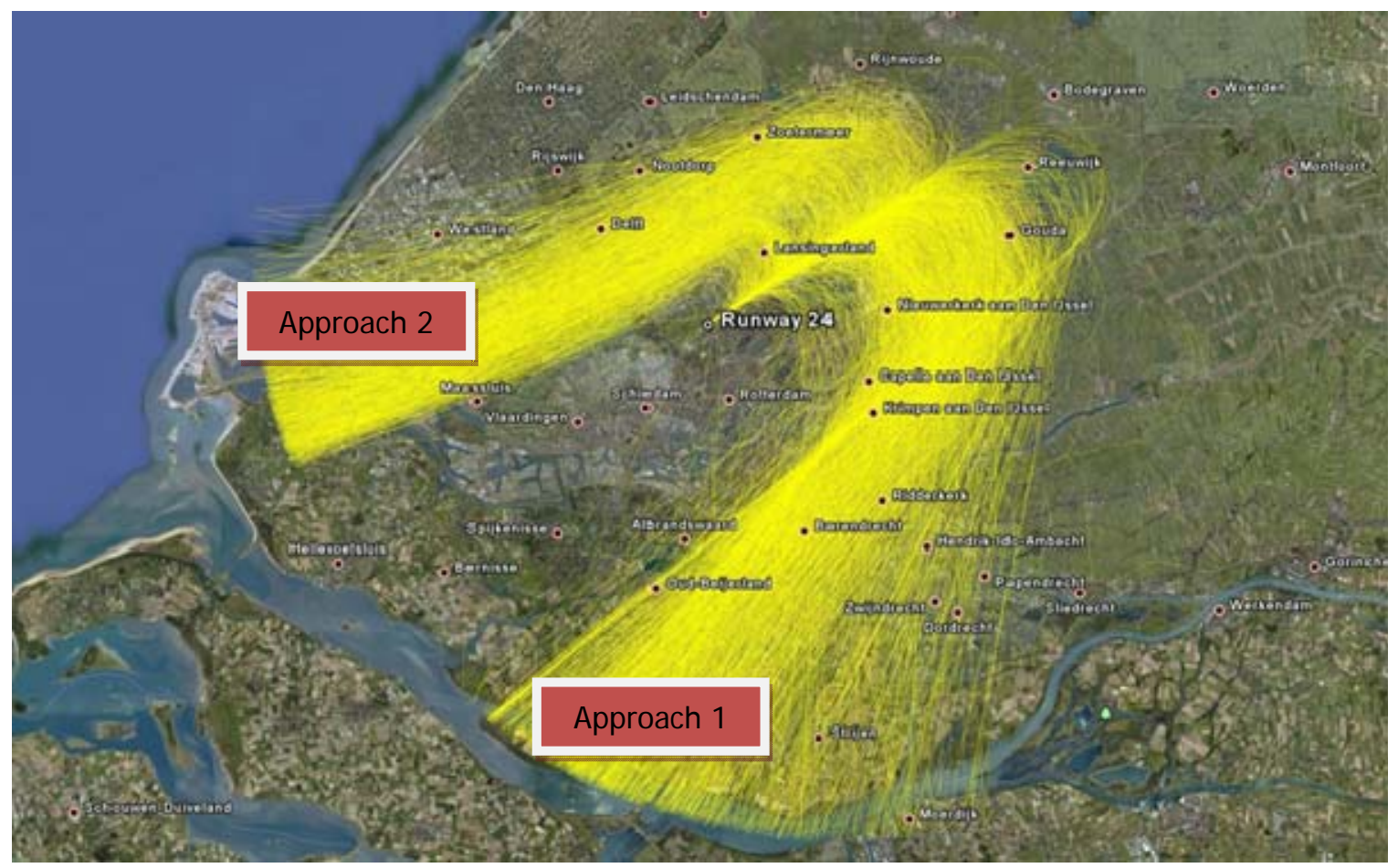

Figure 6. Radar track data to runway 24 for approaches from the South and South-West (non-RNAV benchmark scenario). 
As indicated earlier, for the adapted version of NOISHHH it is necessary to predefine the altitude and speed profiles. The guidelines for noise calculations in the Netherlands ${ }^{14}$ state the altitude and speed profiles for each of the aircraft categories that are to be used for the noise calculations. The attitude profile common for all three aircraft types (left) and the speed profile for the three acoustically representative aircraft types (right), are shown in Figure 7.
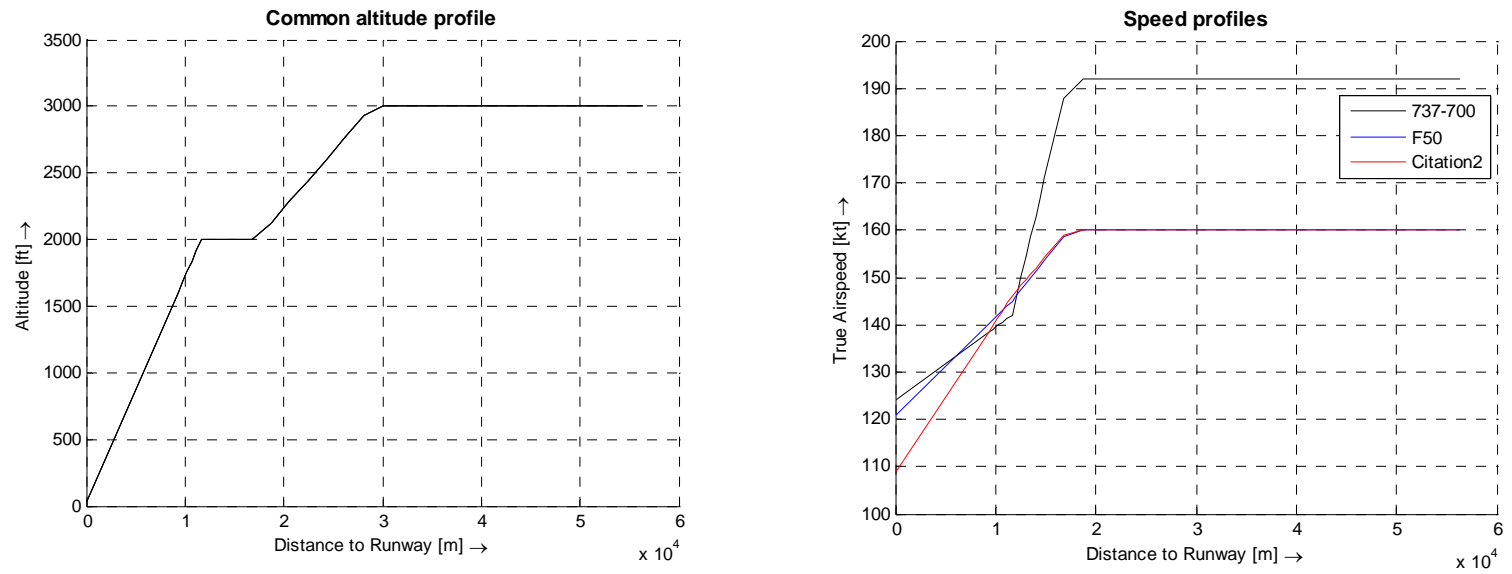

Figure 7. Altitude and speed profiles for the three considered aircraft types.

By combining the standard vertical profiles of the three representative aircraft types with their respective ground tracks shown in Figure 6, the noise contours can be synthesized. Figure 8 shows the noise contours assembled from the accumulated arrivals from the Southern direction, ignoring all other inbound/outbound movements (left subfigure), along with the spatial distribution of the highly annoyed people (right subfigure). It is noted that in the acoustic calculations standard atmospheric conditions have been assumed for all flights. Also, for each considered aircraft type a specific flap extension schedule has been assumed, which specifies the airspeeds at which the flaps are extracted ${ }^{17}$. Increasing the flap setting increases the aircraft drag which in turn leads to a higher required thrust level. This obviously has an adverse effect on the noise footprint and therefore has to be accounted for. The time at which each aircraft arrives at the airport has also been included in the analysis as this determines which penalty factor $w(i)$ is to be applied in the $L_{d e n}$ formula (Eq. 6) for each flight. Using the arrival times included in the radar track data set, the effective amount of arrivals can be determined. For example, a Boeing 737-700 aircraft landing at 23:00 hrs will have a penalty factor of 10 and therefore counts as 10 arrivals in the $L_{d e n}$ formula. Although the actual number of arrivals for the Southern approach is 1627, the effective number of arrival flight is 2925 for this approach. Well over $80 \%$ of the arrivals concern Boeing 737-700 operations. The 2009 non-RNAV traffic scenario, as illustrated in Figure 8, serves as the reference scenario (benchmark) in this study, against which the RNAV trajectory solutions can be compared.

Using the modified NOISHHH tool, a new RNAV Southern approach route has been synthesized. In this synthesis all arrivals from the South to runway 24 as shown in Figure 2 are now assumed to fly a single noise optimal RNAV route. The synthesis of an optimal RNAV route proceeds in two steps. First, an optimal trajectory is generated without explicitly taking into account any RNAV constraints. Based on the observed behavior of the nonRNAV solution, a meaningful route structure then needs to be inferred in terms of a sequence of RNAV segments. In a second step, the trajectory is then re-optimized whilst imposing the RNAV segment pattern identified in the previous step. It is noted that only the sequence of TF and RF legs needs to be specified. Indeed, the length of each $\mathrm{TF}$ leg as well as the circular arc and turn radius of each RF leg (and thus the location of the waypoints) is completely determined in the trajectory optimization process.

Figure 9 shows the noise optimal non-RNAV and RNAV approach routes from the South on a map. The performance index corresponding to this solution is specified such that in the optimization process the primary focus is on noise abatement (i.e., $k_{\text {noise }}=9$ and $k_{\text {distance }}=1$ in Eq.(8)). It needs to be noted that the initial point of a trajectory is assumed to be located on a circle segment with a $35 \mathrm{~km}$ radius centered on the final approach fix (FAF). The RNAV route structure assumed for the RNAV solution in Figure 9 comprises five segments, viz. three TF legs 
alternated by two RF legs. A comparison of the non-RNAV and RNAV solutions in Figure 9 shows that the two trajectories are not truly in close agreement, resulting in a significant performance penalty.
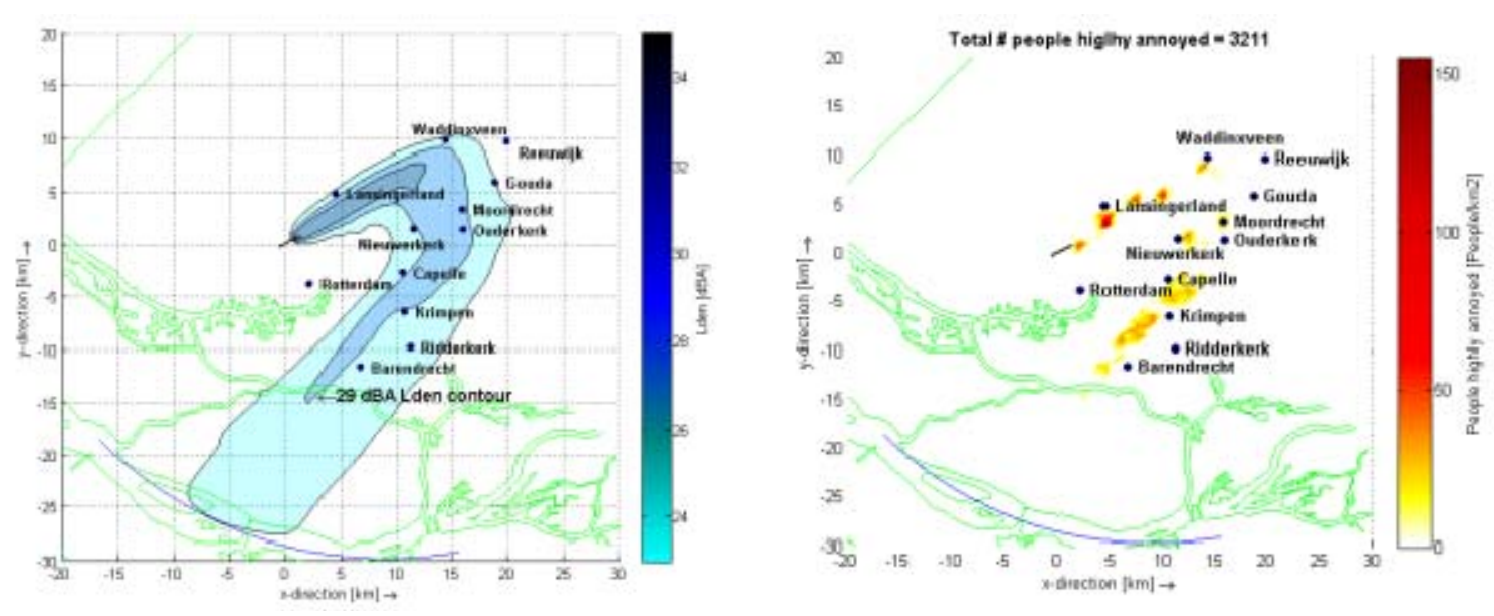

Figure 8. Noise contours $\left(L_{d e n}\right)$ and spatial distribution of the people highly annoyed $(P H)$ for approach 1 in reference scenario.
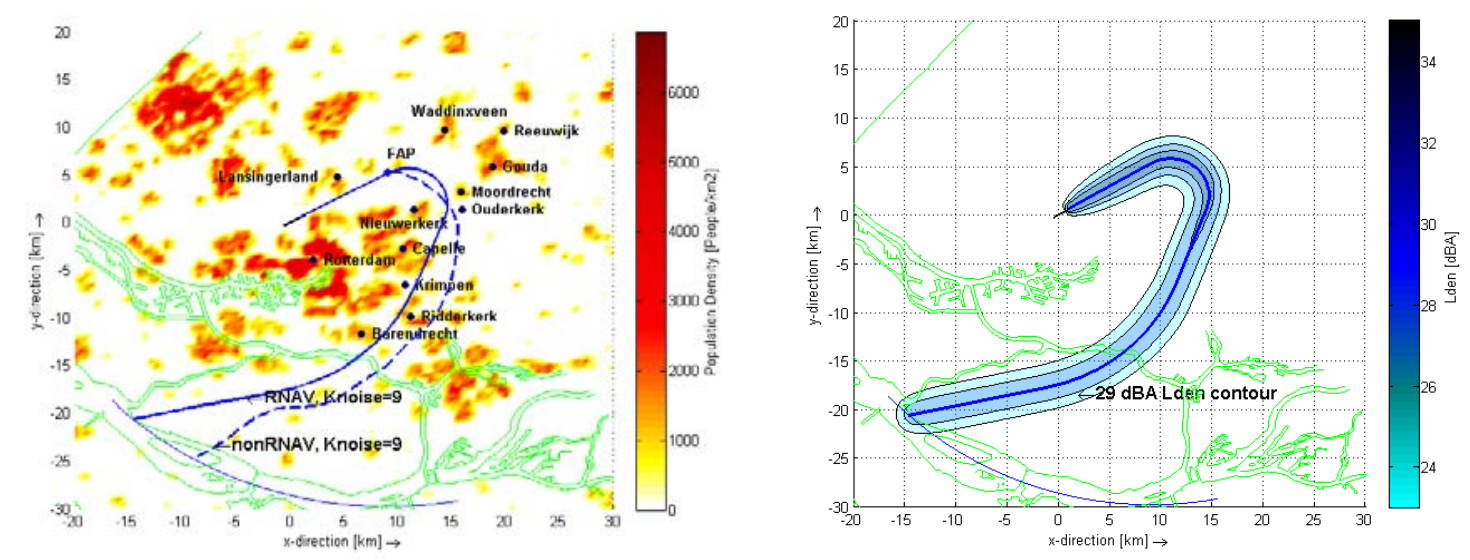

Figure 9. Comparison of non-RNAV and five-segment RNAV optimal solutions (left); Noise contours $\left(L_{d e n}\right)$ for five-segment RNAV optimal solution (right).

In Figure 10, an RNAV solution is shown which features an RNAV structure comprising seven segments, viz. four TF legs alternated by three RF legs. Although this obviously results in a more complicated route, the RNAV solution now closely resembles the non-RNAV solution. The major results are summarized in Table 1. It is recalled that the reference solution (benchmark) corresponds to the current (non-RNAV) situation as shown in Figure 6. The seven-segment optimal RNAV approach reveals that a 30\% decrease in the number of people highly annoyed can be achieved at the expense of a mere $8 \%$ increase in route length compared with current operations (average path length for vectored operations). The results for the seven-segment problem clearly illustrate the impact of increasing the "optimization freedom" on the results. In this case, adding just one turn to the modeling of the approach has led to an additional $10 \%$ reduction in the amount of people highly annoyed relative to the five-segment solution. 

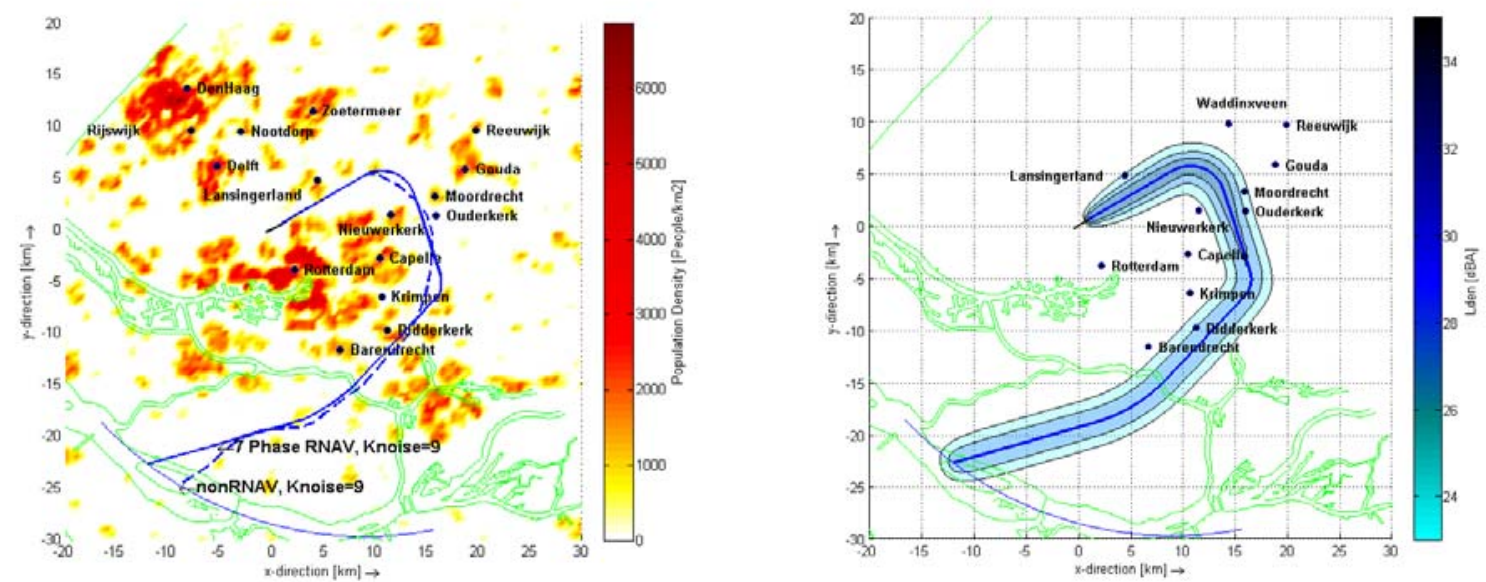

Figure 10. Comparison of non-RNAV and seven-segment RNAV optimal solutions (left); Noise contours $\left(L_{d e n}\right)$ for and seven-segment RNAV optimal solution (right).

Although the concentration of all Southern inbound flights onto a single optimized RNAV route turns out to result in a significant reduction of the expected number of people highly annoyed, it is quite conceivable that spreading the annual inbound traffic over multiple (Southern) RNAV routes may result in an even better noise impact performance. This is a topic for future research.

Table1. Performance of five-segment RNAV and seven-segment RNAV solutions measured against the benchmark..

\begin{tabular}{|l|l|r|}
\hline \multicolumn{2}{|c|}{ Results approach 1, 7 phase RNAV, Knoise=9, Kdistance=1 } \\
\hline \multirow{4}{*}{ Input } & \# effective arrivals CAT 75 [-] & 2547 \\
\hline & \# effective arrivals CAT 71 [-] & 48 \\
\cline { 2 - 3 } & \# effective arrivals CAT 70 [-] & 330 \\
\hline \multirow{3}{*}{ Output Benchmark } & & $\mathbf{3 2 1 1}$ \\
\hline & \# people highly annoyed [-] & $\mathbf{5 4 8 3 3}$ \\
\cline { 2 - 3 } & Average track length [m] & $\mathbf{2 2 5 1}$ \\
\hline \multirow{3}{*}{$\begin{array}{l}\text { Output Optimization } \\
\text { RNAV phase }\end{array}$} & \# people highly annoyed [-] & $\mathbf{5 8 9 9 7}$ \\
\cline { 2 - 3 } & track lenth [m] & $\mathbf{- 3 0}$ \\
\cline { 2 - 3 } & Change in \# people highly annoyed [\%] \\
\cline { 2 - 3 } & Change in track length [\%] & $\mathbf{8}$ \\
\hline \multirow{3}{*}{$\begin{array}{l}\text { Output Optimization } \\
\text { RNAV phase }\end{array}$} & \# people highly annoyed [-] & $\mathbf{5 7 7 0 3}$ \\
\cline { 2 - 3 } & track lenth [m] & -20 \\
\cline { 2 - 3 } & Change in \# people highly annoyed [\%] \\
\cline { 2 - 3 } & Change in track length [\%] & 5 \\
\hline
\end{tabular}




\section{Conclusion}

Through the course of this research, we have developed a novel tool for synthesizing RNAV approach trajectories that minimize a cumulative noise criterion that takes into account the total noise effect of all flight movements over a specified period. This new development is based on an extension of a single-event tool called NOISHHH which was developed earlier for the analysis and design of noise abatement procedures around airports. An important conclusion of this research is that the adapted version of NOISHHH is capable of optimizing approach routes with respect to noise and track length, taking into account the multiple aircraft types that are present in the traffic mix. Moreover, the tool is capable of handling the operational and safety regulations that apply to approach routes in general and to RNAV routes in particular. It proved to be possible to define these constraints in such a way, that the optimized route can be flown by all the aircraft types considered in the analysis.

Application of the adapted NOISHHH framework to the optimization of approach operations at Rotterdam The Hague Airport has led to some very interesting results. Without including RNAV constraints, an improvement of $49 \%$ in terms of people highly annoyed has been achieved with respect to the benchmark situation, with a mere $3 \%$ increase in route length. With RNAV constraints included, still a reduction of $30 \%$ in the number of people highly annoyed could be achieved at the expense of only an $8 \%$ increase in route length. Although no conclusive data has been found regarding the "monetary cost" that can be attached to people highly annoyed, it seems fair to assume that the cost related to the increase in arrival route length (and thus fuel consumption) seen in the various noise optimal solutions generated in this study, is modest compared to the achieved reduction in the number of people highly annoyed.

The use of RNAV routes implies that approach flights will be executed without any significant ground track dispersion. Although our research bears out that introducing noise optimized RNAV routes has the potential to reduce the number of people highly annoyed relative to the present situation, it is readily clear that the population living directly underneath the RNAV flight path is likely to experience a significant increase in noise exposure relative to the existing situation. The justification of introducing RNAV routes from an environmental perspective therefore still remains an open-issue. It is conceivable that spreading the annual arrivals over multiple adjacent RNAV routes will provide relief to the most severely exposed individuals, while simultaneously reducing the overall number of highly exposed individuals. This issue will be explored in future research.

\section{References}

${ }^{1}$ Visser, H.G., Wijnen, R.A.A., "Optimization of Noise Abatement Departure Trajectories,” Journal of Aircraft, Vol. 38, No. 4, 2001, pp. 620-627.

${ }^{2}$ Visser, H.G., Wijnen, R.A.A., "Optimisation of Noise Abatement Arrival Trajectories," The Aeronautical Journal, Vol.107, No. 1076, 2003, pp. 607-615.

${ }^{3}$ Visser, H.G., "Generic and Site-Specific Criteria in the Optimization of Noise Abatement Trajectories," Transportation Research Part D: Transport and Environment, Volume 10, Issue 5, 2005, pp. 405-419.

${ }^{4}$ Hartjes, S., Visser, H.G., and Hebly, S. "Optimisation of RNAV Noise and Emission Abatement Standard Instrument Departures," Aeronautical Journal, Vol.114, No. 1162, 2010, pp. 757-767.

${ }^{5}$ Hogenhuis, ${ }^{6}$ R.H. Heblij, S.J. and Visser, H.G. "Optimization of RNAV Noise Abatement Arrival Trajectories," Proceedings of the Institution of Mechanical Engineers - Part G - Journal of Aerospace Engineering, Vol. 225, Issue 5, 2011, pp.513-521 .

${ }^{6}$ Prats, X., Puig, V., Quevedo, J., and Nejjari, F., “ Lexicographic optimisation for optimal departure aircraft trajectories," Aerosp. Sci. Technol., Vol.14, Issue 1, 2010, pp. 26-37.

${ }^{7}$ Torres, R., Chaptal, J, Bès, C., and Hiriart-Urruty, J-B., "Optimal, Environmentally-Friendly Departure Procedures for Civil Aircraft," Journal of Aircraft, Vol 48, No 1, 2011, pp. 11-22.

${ }^{8}$ Zaporozhets,O. I., and Tokarev,V. I., "Predicted flight procedures for minimum noise impact," J.Appl.Acoust., Vol. 55, No.2, 1998, pp.129-143.

${ }^{9}$ Zachary, D.S., Gervais, J., Leopold, U., Schutz, G., Huck,.S.T., and Braun, C. "Strategic planning of aircraft movements with a three-cost objective," Journal of Aircraft, Vol. 48, No. 1, January 2011, pp.256-264 .

${ }^{10}$ Zachary, D.S., Gervais, J., and Leopold, U., "Multi-impact optimization to reduce aviation noise and emissions," Transportation Research Part D: Transport and Environment, Vol. 15, Issue 2, March 2010, pp.82-93. 
${ }^{11}$ Hebly, S.J., Hanenburg, V.A., Wijnen, R.A.A., and Visser, H.G., "Development of a Noise Allocation Planning Tool," Proceedings of the 45th AIAA Aerospace Sciences Meeting and Exhibit, AIAA, Reston, VA, 2007.

${ }^{12}$ Heblij, S. J., and Wijnen, R. A. A., "Development of a runway allocation optimisation model for airport strategic planning," Transportation Planning and Technology, 31(2), 2008, pp. 201-214.

${ }^{13}$ Visser, H.G., "Environmentally Optimized Resolutions of In-Trail Separation Conflicts for Arrival Flights," Journal of Aircraft, Vol. 45, No. 4, July-August 2008, pp. 1198-1205.

${ }^{14}$ De Jong, R., Heppe, G.J.T., "Appendices van de voorschriften voor de berekening van de geluidsbelasting," (in Dutch), Versie 10.1, NLR-CR-96950L, 2007.

${ }^{15}$ Office of Environment and Energy, "Integrated Noise Model (INM) Version 6.0 Technical Manual," Rept. FAA-AEE-02-01, U.S.A., 2002.

${ }^{16}$ Jabben, J., Verheijen, E., Schreurs, E., "Groepsgeluidbelasting Gden/Gnight : Toepassingsmogelijkheden luchtvaartgeluid," (in Dutch), RIVM Briefrapport 680555004/2010, 2010.

${ }^{17}$ Braakenburg, M.L., "Development of a multi-event optimization tool for noise optimized arrival route design," MSc thesis, TU Delft, November 2010. 